\title{
NaD excess objects and its implications on initial mass function
}

\author{
Sukyoung K. Yi ${ }^{1}$ and Hyunjin Jeong ${ }^{2}$ \\ ${ }^{1}$ Yonsei University, Astronomy Department, Seoul 120-749, Republic of Korea. email: \\ yi@yonsei.ac.kr \\ ${ }^{2}$ Korea Astronomy and Space Science Institute Daejeon 305-348, Republic of Korea. email: \\ hyunjin@kasi.re.kr
}

\begin{abstract}
Dokkum and Conroy (2010) have heralded the possible implication of NaI 8200A lines on extra bottom-heavy IMF of bright elliptical galaxies. A flurry of papers have followed it and revisited the half a century old issue all over again from various angles. For a sanity check, we investigate on the issue this time using the better measured NaD 5900A line instead. We found from the SDSS database that a large $(\sim 10 \%)$ fraction of galaxies (both early and late types) exhibit strong $\mathrm{NaD}$ lines and that the same bottom-heavy models of van Dokkum and Conroy that reproduced the strong NaI 8200 lines of elliptical galaxies are not capable of reproducing $\mathrm{NaD}$ line strengths at all. The mystery prevails. This article is mainly a review of our recent paper (Jeong et al. 2013).
\end{abstract}

Keywords. galaxies: elliptical and lenticular, cD - galaxies: evolution - galaxies: formation

\section{Introduction}

The fact that the apparently boring spectra of elliptical galaxies have many hints to their formation history is evident and it was properly highlighted by van Dokkum and Conroy through their series of papers (van Dokkum and Conroy 2010; 2011; 2012). Na lines (mostly NaD 5900) have been particularly of interest ever since their strengths were noticed to be much stronger in bright elliptical galaxies than expected for "normal?? stellar populations (O?Connell 1976; Peterson 1976; Hardy and Couture 1988; Worthey 1988).

van Dokkum and Conroy in their series of papers reported that the NaI 8200 line strengths in the near infrared are also very strong in bright ellipticals and that such strong line strengths can be explained if the bulk of the stellar populations in them are even more bottom heavy than the Salpeter IMF which is generally believed to be already more bottom heavy than the reality in most environments. This obviously provoked the community greatly resulting in a cascade of many papers on the issue (e.g., Spiniello et al. 2011; Worthey et al. 2011; Capellari et al. 2012; Ferreras et al. 2013; Smith 2014).

IMF has been one of those scary contents in the Pandora chest of astronomy along with black holes, dark matter, binaries, and turbulence. Whenever we are incapable of explaining something we are tempted to open the chest and see if any of the contents can help solve the problem. But this is usually the final choice where nothing else can be done, because, although their presence is not doubted, their abundance and effects are not clearly understood at all while their side effects are formidable.

van Dokkum and Conroy used mainly NaI 8200 in their investigation. It is in a tough region of wavelength for measurement because telluric contamination is difficult to remove. NaD 5900 is on the other hand much stronger and easy to measure being in the optical band. A large spectroscopic survey such as SDSS provides its strength for millions of galaxies. So, we have performed a sanity check whether the same models that 
were used to match the $\mathrm{NaI} 8200$ of bright ellipticals can also reproduce $\mathrm{NaD} 5900$ on the same types of galaxies. Much of the result of our investigation has already been published (Jeong et al. 2013) and readers are asked to visit the paper for further details.

\section{NaD Excess Objects (NEOs)}

We found many bright galaxies do show strong NaD 5900 lines from SDSS. We first constructed a volume-limited sample $\left(z<0.08, M_{r}<-20.5, \mathrm{~S} / \mathrm{N} ; 20\right)$ of galaxies and secured a better subset of data using the spectral fitting quality assessment of the OSSY database (Oh et al. 2011). OSSY provides new and improved measurements of all stellar absorption and emission lines of SDSS galaxies. We found that 1603 galaxies $(7.8 \%$ of all these bright galaxies) show strong $\mathrm{NaD}$ lines and thus were named ?NaD Excess Objects? (NEOs). We were able to morphological classify 963 of them (others were very uncertain) into 553 early-type and 410 late-type galaxies.

\section{Late-type NEOs}

Late-type NEOs are not an issue here and so are only briefly summarized as follows. Compared to the control sample, most late-type NEOs seem more actively forming stars and dusty. Their NaD strengths are too strong to be of stellar origin, and it is natural to guess that they are of non-stellar origin, for example, due to interstellar matter processes. Similar conclusions were reported by Chen et al. (2010).

\section{Early-type NEOs}

We found it necessary to separate early-type NEOs into (morphologically-)peculiar NEOs and (morphologically-)smooth NEOs because their properties were significantly different from each other.

\subsection{Morphologically-peculiar early-type NEOs}

First on the peculiar NEOs. They often show merger-related features or dust lanes. Compared to their control sample (of ordinary non-NEO elliptical galaxies) they appear to be flatter and dustier, that can be a hint of disky components. They occupy a wide range of stellar mass with no clear preference of mass. As can be guessed from their dusty nature, they show star-formation hinting (by BPT diagnostics) emission lines much more frequently than the control sample does. Their Balmer line based (and thus luminosityweighted) ages are much younger (roughly $3 \mathrm{Gyr}$ ) compared to those of the control sample (roughly 10Gyr). Just like late-type NEOs, their NaD strengths go outside the grids of stellar population models. In essence, peculiar early-type NEOs are much like late-type NEOs. And it is not unnatural to suspect a non-stellar origin for their strong NaD lines.

\subsection{Morphologically-smooth early-type NEOs}

These are the ones that are similar to the galaxies studied by van Dokkum and Conroy and hence of interest. Compared to the control sample, they look normal in every sense. There is no peculiarity in morphology. They look round and the internal extinction (adopted from OSSY) appears negligibly low. There is strong mass effect: the more massive the stronger $\mathrm{NaD}$ absorption line, in good agreement with van Dokkum and Conroy (2012). Only a small fraction ( $₫ 10 \%)$ shows emission lines, but even then, most of them indicate LINER origin rather than star formation. Their integrated colours are redder. The absorption line analysis suggests super solar in metals and alpha elements. 
Their luminosity-weighted ages are as old as those of the control sample. Our new analysis shows no notable doppler signature on the $\mathrm{NaD}$ lines, indicating no inflow-outflow related origins (Park, Jeong and Yi in prep.). In every sense, they are showing stellar lights of typical old populations. Therefore, the strong Na lines should also be of stellar origin.

\section{Origin of smooth early-type NEOs}

We now focus only on smooth-looking early-type NEOs. Figure 21 of Jeong et al. (2013) sums up the situation as to whether the current models can reproduce the observed strengths of NaD lines of smooth early-type NEOs. From our absorption line analysis, it seems almost certain that smooth early-type NEOs are more metal rich in Fe and alpha elements probably by as much as $[\mathrm{Fe} / \mathrm{H}] \sim+0.3$ and $[\alpha / \mathrm{Fe}] \sim+0.3$. Both of these overabundances make $\mathrm{NaD}$ lines stronger but not as much as found in smooth earlytype NEOs. We need an extra enhancement in $\mathrm{Na}$ by as much as $[\mathrm{Na} / \mathrm{Fe}] \sim+0.3$ to fully account for the strengths of $\mathrm{NaD}$ lines observed. The use of bottom heavy IMF hardly helps the situation. It enhances $\mathrm{NaD}$ line strength but almost insignificantly, even if we use Conroy?s models. As a result, the rescue plan of bottom heavy IMF that was proposed to solve the mystery of strong NaI 8200 seems futile when it comes to $\mathrm{NaD}$ 5900 .

\section{Discussion}

At this conference, Conroy presented a talk and also confirmed publicly that the use of bottom heavy models did not reproduce NaD line strengths. Only with an additional and $a d$ hoc $\mathrm{Na}$ enhancement of $[\mathrm{Na} / \mathrm{Fe}] \sim+0.3$ was he able to reproduce the observation, which is consistent with what we have found. We are pleased with this agreement, but that means that the enigma of $\mathrm{Na}$ line excess is still an outstanding issue. There is little doubt that $\mathrm{Na}$ line strengths contain critical information on IMF and other details of galaxy formation, but whether they are truly indicative of extra bottom-heavy IMF as originally proposed is doubtful.

\section{References}

Chen, Y.-M., Tremonti, C. A., Heckman, T., Kauffmann, G., Weiner, B., Brinchmann, J., \& Wang, J., 2010, AJ, 140, 445

Dutton, A. A., Maccio, A. V., Mendel, J. T., \& Simard, L., 2013, MNRAS, 432, 2496

Ferreras, I., La Barbera, F., de la Rosa, I. G., Vazdekis, A., de Carvalho, R. R., Falcon-Barroso, J., \& Ricciardelli, E., 2013, MNRAS, 429, 15

Hardy, E. \& Coutour, J., 1988, ApJ, 325, 29

Jeong, H., Yi, S. K., Kyeong, J., Sarzi, M., Sung, E.-C., \& Oh, K., 2013, ApJS, 280, 7

Oh, K., Sarzi, M., Schawinski, K., \& Yi, S. K., 2011, ApJS, 195, 13

O'Connell, R. W., 1976, ApJ, 206, 370

Peterson, R., 1976, ApJ, 210, 123

Smith, R., 2014, MNRAS, 443, 69

van Dokkum, P. \& Conroy, C., 2010, Nature, 468, 940

van Dokkum, P. \& Conroy, C., 2011, ApJ, 735, 13

van Dokkum, P. \& Conroy, C., 2012, ApJ, 760, 70

Worthey, G., Ingermann, B. A., \& Serven, J., 2011, ApJ, 729, 148 